\title{
MicroRNAs: A New Class of Master Regulators of Adipogenesis
}

\section{Carlo Mercado, Gabriel Eades and Qun Zhou*}

Department of Biochemistry and Molecular Biology, University of Maryland School of Medicine, Baltimore, MD 21201, USA

\begin{abstract}
Adipogenesis is the process by which Mesenchymal Stem cells commit to become adipocyte precursor cells, which will later differentiate into mature adipocytes following exposure to differentiation factors. The transcriptional programs involved in the differentiation process have been carefully studied. Recently, small non-coding RNAs, microRNAs, have been found to play critical roles throughout the adipogenic process. MicroRNAs have been identified that are involved in promoting or inhibiting adipogenesis by targeting anti- or pro-adipogenic factors and cell cycle regulatory proteins. Here, we will discuss the latest discoveries regarding microRNA regulation of adipocyte differentiation.
\end{abstract}

Keywords: Adipogenesis; Mesenchymal stem cells; MicroRNAs; Adipocyte differentiation

\section{Function of Adipose Tissue}

Adipose tissue, or body fat, is an essential class of connective tissue prominent throughout the body. Adipose tissue serves to regulate and maintain energy homeostasis within an organism, and is primarily composed of adipocytes, or fat cells [1]. These cells are classified into two distinct types: White Adipose Tissue (WAT) and Brown Adipose Tissue (BAT). WAT is an active endocrine organ whose function is to store energy and to regulate the activity of insulin, lipid metabolism and satiety. BAT is located in discrete pockets, contains a large number of mitochondria and undergoes thermogenesis, where heat is expelled as chemical energy [2]. This review mainly focuses on the impact of microRNAs on WAT-related adipogenesis.

Adipocytes function to store reserves of nourishment in times of abundance, and to release these reserves in times of need. This ability of adipose tissue to maintain the necessary amount of energy for use in metabolism is essential for the survival of any organism. However, the intake of too much fat may result in dysfunctional adipose tissue, which may contribute to disease development including obesity, diabetes, and coronary artery disease [3,4]. Indeed, adipocytes are much larger in size in obese individuals compared to those in leaner counterparts [5]. As such, a thorough assessment on the regulation of adipocyte differentiation may provide a better understanding on the molecular mechanisms that give rise to adipogenic dysfunctions. These mechanisms could potentially serve as markers for therapy against metabolic disorders.

\section{Adipogenesis}

Adipocytes differentiate from mesenchymal stem cells (MSCs) in a process known as adipogenesis, and normally surround themselves with a network of other cell-types that include fibroblasts, preadipocytic cells, endothelial cells, nerves and immune cells [6]. Although much of the classic knowledge regarding adipogenic regulation delves into the existence of various regulatory proteins, recent literature has touched upon the role of microRNAs in the differentiation of MSCs to adipocytes. This review summarizes the current paradigms that support an association between numerous regulatory microRNAs and the adipogenic process.

During adipogenesis, cells from adipose tissue proliferate and differentiate into lipid assimilating cells. This is known to occur in two distinct stages: the first stage being the commitment stage, in which MSCs commit to becoming preadipocytes, and the second stage being the terminal differentiation stage [7]. During adipogenic competency and commitment, extracellular matrix (ECM) and cell shape remodeling play a role in coordinating various signaling cascades [2]. This stage involves many key regulators that are modulated during the transition between MSC to preadipocytes, including WNT family members and Rho-family GTPases [2]. WNT family members are secreted glycoproteins that are known to inhibit adipogenesis through WNT ligand-mediated signaling cascades. This WNT signaling activity is not exhibited in mouse embryonic fibroblasts lacking the WNT receptor, which in turn develop increased adipocyte differentiation $[8,9]$.

\section{Adipogenic Commitment}

MSC commitment to preadipocytes is characterized by ECM stiffness that results in tension, which enhances actin and myosin formation and subsequent cell stretching $[7,10]$. This phenomenon was previously observed in 3T3-F442A cells, in which differentiation into adipocytes was inhibited by fibronectin and rescued by chemical inhibition of actin stress fiber formation [11]. Furthermore, both human MSCs and mouse preadipocytes grown on stiffer matrices with higher concentration of collagen I display reduced rates of adipocyte differentiation than those grown in softer gels with lower stiffness $[12,13]$.

Ectopic induction of a5 integrin inhibits adipocyte differentiation by maintaining high expression of the Rho family GTPase known as Rac in its activated form (Rac bound to GTP) [14]. Rac-GTP activates Rho GTPase-Rho-associated kinase (ROCK) signaling, which facilitates actinomyosin fiber formation that blocks adipogenesis $[15,16]$. In order for differentiation to occur, Rac-GTP must be down-regulated and its GDP bound form (inactive) must predominate. This is experimentally shown in 3T3-L1 cells treated with adipogenic stimuli, in which a5

${ }^{*}$ Corresponding author: Qun Zhou, Department of Biochemistry and Molecula Biology, University of Maryland School of Medicine, Baltimore, MD 21201, USA Tel: 410-706-1615; Fax: 410-706-8297; E-mail: qzhou@som.umaryland.edu

Received April 23, 2013; Accepted May 02, 2013; Published May 04, 2013 Citation: Mercado C, Eades G, Zhou Q (2013) MicroRNAs: A New Class of Master Regulators of Adipogenesis. Human Genet Embryol 3: 108. doi:10.4172/21610436.1000108

Copyright: (c) 2013 Mercado C, et al. This is an open-access article distributed under the terms of the Creative Commons Attribution License, which permits unrestricted use, distribution, and reproduction in any medium, provided the original author and source are credited. 
integrin is repressed, Rac is inactivated and terminal differentiation commences [14].

Cell-to-cell contact and cell shape are also factors that can influence adipogenesis. Preadipocytes undergo a period of mitotic clonal expansion upon the addition of adipogenic stimuli such as cAMP agonist, insulin and glucocorticoids. These stimuli further advance MSCs to peadipocytic commitment and committed preadipoctyes to confluency and a rounded morphology $[2,17,18]$. Differentiation of confluent preadipocytes requires cell cycle regulatory proteins such as E2Fs and p130 [19]. Once the preadipocytes are fully committed, a signal cascade is activated to induce adipocyte differentiation.

\section{Terminal Differentiation of Adipocytes}

Terminal differentiation begins with the expression of two families of genes that encode the regulatory proteins CCAAT/enhancer binding protein $(\mathrm{C} / \mathrm{EBP})$ and the adipogenic master regulator, Peroxisome proliferator-activated receptor $(\mathrm{PPAR} \gamma)$. Adipogenic stimuli promote the down-regulation of adipogenesis repressor preadipocyte factor 1 (Pref-1) and the increased expression of $\mathrm{C} / \mathrm{EBP} \beta$ and $\mathrm{C} / \mathrm{EBP} \delta$, which together recruit a transcriptional activation complex that consists of transcription factors, glucocorticoid receptor, STAT5A, RXR and a co-activator complex [2]. This complex recognizes acetylated and methylated regions of the PPARG locus that are epigenetically marked for histone modification [20-22]. Previous studies have reported on the conservation of adipogenic stimulus-dependent induction of PPAR $\gamma$ binding and histone modification near genes involved in adipogenesis, such as fatty acid binding protein 4 (FABP4), PPARG and GLUT4 [2, 22].

C/EBPß binds to DNA when phosphorylated by the MAPK pathway and glycogen synthase kinase $3 ß$ and synergizes with C/EBP $\delta$ to activate transcription of PPAR $\gamma$ [23-25]. During terminal differentiation, a shift in gene expression occurs between genes encoding transcription factors that are prominent in the preadipocyte phenotype and those that are prominent in the adipocyte phenotype. C/EBPa and PPAR $\gamma$ work in tandem to mediate the expression of these adipogenic gene products, which include FABP4, adipsin and lipoprotein lipase (LPL) [2, 26, 27].

Additional adipogenic transcription factors include Krüppellike factors (KLFs) and C10orf116. The latter is a novel transcription factor that has recently been discovered to transcribe the protein, Adipogenesis Factor Rich in Obesity (AFRO), which is thought to stimulate the transcription of PPAR $\gamma$ and $\mathrm{C} / \mathrm{EBPa}$ in the early stages of adipogenesis [25]. KLFs are zinc finger transcription factors that serve as both positive and negative adipogenic regulators, depending on the isoform. For example, KLF5, a positive adipogenic regulator, works in concert with $\mathrm{C} / \mathrm{EBP} \delta$ to induce the expression of PPAR $\gamma 2[28,29]$.

\section{MicroRNA Regulation of Adipogenesis}

Current findings report on the existence of numerous inhibitory and promoting microRNAs (miRs) involved in the regulation of adipogenesis, both in the commitment stage and in terminal differentiation. MiRs are defined as a set of non-coding RNAs ranging from about 18 to 22 nucleotides in length. Their function is to target specific mRNA transcripts for degradation or translational repression, thus playing a regulatory role in numerous biological processes such as development, reproduction and differentiation [30].

Knelangen et al. conducted a miR profile in mouse embryonic stem cells to monitor miR involvement during the entire process of adipogenic differentiation. From this profile, 129 miRs exhibited change in expression levels at distinct time points, ranging from mesodermal progenitor cells (day 5) to mature adipocytes (day 21) [31].

\section{miR-17-92 cluster}

The miR-17-92 cluster was found to accelerate adipocyte differentiation by inhibiting the tumor suppressor, $\mathrm{Rb} 2 / \mathrm{p} 130$ during early clonal expansion of preadipocytes. The miR-17-92 cluster is abundantly expressed in embryonic stem cells (ESCs) where researchers believe that this miR cluster gets sequestered with alternative networks to maintain the pluripotency and self-renewal associated with an ESC state $[31,32]$.

\section{miR-138}

MiR-138 knocks down the expression of EID1, which is a gene involved in adipocyte differentiation in adipose-derived MSCs. Previous research has shown that overexpression of miR-138 reduces triglyceride accumulation and inhibits the expression of adipogenic markers such as C/EBPa and PPAR $\gamma$ [33].

\section{miR-155}

Terminal differentiation from preadipocytes to mature adipocytes is also governed by miRs [31]. For example, tumor necrosis factor (TNFa)-mediated overexpression of miR-155 via the NFkB pathway results in the down-regulation of C/EBPß and CREB [34]. MiR-155 inhibits these proteins by targeting their 3 'untranslated regions (UTRs), thereby inhibiting the transcription of later adipogenic markers, PPAR $\gamma$ and C/EBPa. This was shown experimentally through the use of an antimiR-155 that reduced TNFa-induced inhibition of adipogenesis and through exogenous expression of miR-155, which inhibits adipogenesis [34].

\section{miR-27 and miR-130}

MiR-27a/b and miR-130 are known adipogenic inhibitors that target PPAR $\gamma$ directly [35-37]. Chan et al. performed analyses on miR-27b in tandem with a component of ginseng extract known as Ginsenoside- $R b_{1}\left(R b_{1}\right)$. This group postulated that $R b_{1}$ might function to down-regulate miR-27b gene transcription and mature miR-27b activity to facilitate PPAR $\gamma$ expression and subsequent adipogenesis [38]. They also found that treating adipocytes with $\mathrm{Rb}_{1}$ resulted in decreased expression of pri-miR-27b as well as mature miR-27b. Furthermore, suppressing PPAR $\gamma$ with the antagonist, GW9662 in $\mathrm{Rb}$ treated adipocytes rescued the expression of mature miR-27b and pri-miR-27b, which supports that inhibition of miR-27b transcription and function is PPAR $\gamma$-dependent [38]. Another study reports on a significant lower level of miR-130 expression and a markedly higher expression of PPAR $\gamma$ mRNA in adipose tissue obtained from obese women compared to that of lean women [37]. These studies reveal that miR-130 potently represses PPAR $\gamma$ expression and biosynthesis through selective targeting of PPAR $\gamma$ mRNA coding as well as its $3^{\prime}$ UTR.

\section{miR-448}

MiR-448 selectively targets KLF5 to down-regulate its induction of PPAR $\gamma$ expression, thereby reducing adipocyte differentiation [39]. This was confirmed by results obtained from 3T3-L1 cells overexpressing miR-448, in which researchers observed reduced triglyceride accumulation. Conversely, researchers observed an increase in adipocyte differentiation in 3T3-L1 cells lacking miR-448 [39].

\section{miR-200 family}

Some examples of pro-adipogenic miRs include members of the 
miR-200 family (mir-200c/141 and miR200b, a/429 clusters), which inhibit WNT singling that normally functions to down-regulate C/ EBP $\alpha$ and PPAR $\gamma[40,41]$.

\section{miR-143}

There have been numerous recent findings regarding the link between regulatory miRs and adipogenesis, including the classification of miR-143 as a "fine-tuning miR" rather than an adipogenic switch. $\mathrm{He}$ et al. reports that Fgf7, a member of the fibroblast growth factor family, is a putative target of miR-143 [30]. Fgfy may function as a fine-tuning molecule in the adipogenic process. He et al. observed that knock down of miR-143 does not yield significant changes in phenotype with in vivo approaches compared to in vitro studies, which may be attributed to redundant functions of other related miRs [30].

\section{miR-140}

Recently, miR-140 was identified as a downstream component of BMP4 signaling that acted to promote adipocyte commitment. MiR-140 was found to directly target Osteopetrosis-associated transmembrane protein 1 (Ostm1), an anti-adipogenic factor [42].

\section{Outstanding Questions}

Recent experimental approaches that manipulate the expression levels of various miRs in cell cultures have resulted in either the inhibition or stimulation of adipocyte differentiation. These analyses support a potential regulatory function for miRs in mammalian adipogenesis, in which miRs selectively target anti- or pro-adipogenic factors. Some major discoveries in the field include the negative regulation of EID1 by miR-138 in MSCs [33]; the link between miR-27b regulation and $\mathrm{Rb}_{1}$ promoting adipogenesis with PPAR $\gamma$ binding [38]; C/EBPß and CREB inhibition as a result of TNF $\alpha$-induced overexpression of miR-155 [34]; and more recently, the classification of miR-143 as an adipogenic finetuner [30]. Since most of these findings rely on in vitro studies, further in vivo analyses are needed to evaluate a defined association between miRs and adipogenesis. Indeed, the scientific community has yet to completely address the complexity of post-transcriptional regulation that miRs perform in adipogenesis. This will require proteomic studies, since adipogenic regulation by miRs may be more apparent at the protein level, as well as further insight on candidate adipogenic markers that are specific for distinct stages of precursor cell development.

\section{References}

1. Galic S, Oakhill JS, Steinberg GR (2010) Adipose tissue as an endocrine organ. Mol Cell Endocrinol 316: 129-139.

2. Cristancho AG, Lazar MA(2011) Forming functional fat: a growing understanding of adipocyte differentiation. Nat Rev Mol Cell Biol 12: 722-734.

3. Poulos SP, Hausman DB, Hausman GJ (2010) The development and endocrine functions of adipose tissue. Mol Cell Endocrinol 323: 20-34.

4. Rosen ED, Walkey CJ, Puigserver P, Spiegelman BM (2000) Transcriptional regulation of adipogenesis. Genes Dev 14: 1293-1307.

5. Duursma AM, Kedde M, Schrier M, le Sage C, Agami R (2008) miR-148 targets human DNMT3b protein coding region. RNA 14: 872-877.

6. Cinti S (2005) The adipose organ. Prostaglandins Leukot Essent Fatty Acids 73: $9-15$

7. Akimoto T, Ushida T, Miyaki S, Akaogi H, Tsuchiya K, et al. (2005) Mechanical stretch inhibits myoblast-to-adipocyte differentiation through Wnt signaling. Biochem Biophys Res Commun 329: 381-385.

8. Takada I, Kouzmenko AP, Kato S (2009) Wnt and PPARgamma signaling in osteoblastogenesis and adipogenesis. Nat Rev Rheumatol 5: 442-447.

9. Kawai M, Mushiake S, Bessho K, Murakami M, Namba N, et al. (2007) Wnt/Lrp/ beta-catenin signaling suppresses adipogenesis by inhibiting mutual activation of PPARgamma and C/EBPalpha. Biochem Biophys Res Commun 363: 276 282.

10. Jakkaraju S, Zhe X, Pan D, Choudhury R, Schuger L (2005) TIPs are tensionresponsive proteins involved in myogenic versus adipogenic differentiation. Dev Cell 9: 39-49.

11. Spiegelman BM, Ginty CA (1983) Fibronectin modulation of cell shape and lipogenic gene expression in 3T3-adipocytes. Cell 35: 657-666.

12. Winer JP, Janmey PA, McCormick ME, Funaki M (2009) Bone marrow-derived human mesenchymal stem cells become quiescent on soft substrates but remain responsive to chemical or mechanical stimuli. Tissue Eng Part A 15 147-154.

13. Chun TH, Hotary KB, Sabeh F, Saltiel AR, Allen ED, et al. (2006) A pericellular collagenase directs the 3-dimensional development of white adipose tissue. Cell 125: 577-591.

14. Liu J, DeYoung SM, Zhang M, Zhang M, Cheng A, et al. (2005) Changes in integrin expression during adipocyte differentiation. Cell Metab 2: 165-177.

15. McBeath R, Pirone DM, Nelson CM, Bhadriraju K, Chen CS (2004) Cell shape, cytoskeletal tension, and RhoA regulate stem cell lineage commitment. Dev Cell 6: 483-495.

16. Kilian KA, Bugarija B, Lahn BT, Mrksich M (2010) Geometric cues for directing the differentiation of mesenchymal stem cells. Proc Natl Acad Sci U S A 107 4872-4877.

17. Green H, Meuth M (1974) An established pre-adipose cell line and its differentiation in culture. Cell 3: 127-133.

18. Kuri-Harcuch W, Green H (1978) Adipose conversion of 3 T3 cells depends on a serum factor. Proc Natl Acad Sci U S A 75: 6107-6109.

19. Fajas L, Egler V, Reiter R, Hansen J, Kristiansen K, et al. (2002) The retinoblastoma-histone deacetylase 3 complex inhibits PPARgamma and adipocyte differentiation. Dev Cell 3: 903-910.

20. Steger DJ, Grant GR, Schupp M, Tomaru T, Lefterova MI, et al. (2010) Propagation of adipogenic signals through an epigenomic transition state. Genes Dev 24: 1035-1044.

21. Siersbæk R, Nielsen R, John S, Sung MH, Baek S, et al. (2011) Extensive chromatin remodelling and establishment of transcription factor 'hotspots' during early adipogenesis. EMBO J 30: 1459-1472.

22. Mikkelsen TS, Xu Z, Zhang X, Wang L, Gimble JM, et al. (2010) Comparative epigenomic analysis of murine and human adipogenesis. Cell 143: 156-169.

23. Park BH, Qiang L, Farmer SR (2004) Phosphorylation of C/EBPbeta at a consensus extracellular signal-regulated kinase/glycogen synthase kinase 3 site is required for the induction of adiponectin gene expression during the differentiation of mouse fibroblasts into adipocytes. Mol Cell Biol 24: 8671-8680.

24. Tang QQ, Grønborg M, Huang H, Kim JW, Otto TC, et al. (2005) Sequentia phosphorylation of CCAAT enhancer-binding protein beta by MAPK and glycogen synthase kinase 3 beta is required for adipogenesis. Proc Natl Acad Sci U S A 102: 9766-9771.

25. Ni Y, Ji C, Wang B, Qiu J, Wang J, et al. (2012) A Novel pro-adipogenesis factor abundant in adipose tissues and over-expressed in obesity acts upstream of PPAR ${ }^{3}$ and C/EBPÎ士 J Bioenerg Biomembr

26. Farmer SR (2006) Transcriptional control of adipocyte formation. Cell Metab 4: $263-273$.

27. Otto TC, Lane MD (2005) Adipose development: from stem cell to adipocyte. Crit Rev Biochem Mol Biol 40: 229-242.

28. Romao JM, Jin W, Dodson MV, Hausman GJ, Moore SS, et al. (2011) MicroRNA regulation in mammalian adipogenesis. Exp Biol Med (Maywood) 236: 997-1004.

29. Oishi Y, Manabe I, Tobe K, Tsushima K, Shindo T, et al. (2005) Krüppel-like transcription factor KLF5 is a key regulator of adipocyte differentiation. Cell Metab 1: 27-39.

30. He Z, Yu J, Zhou C, Ren G, Cong P, et al. (2013) MiR-143 is not essential for adipose development as revealed by in vivo antisense targeting. Biotechnol Lett 35: 499-507.

31. Knelangen JM, van der Hoek MB, Kong WC, Owens JA, Fischer B, et al 
Citation: Mercado C, Eades G, Zhou Q (2013) MicroRNAs: A New Class of Master Regulators of Adipogenesis. Human Genet Embryol 3: 108. doi:10.4172/2161-0436.1000108

(2011) MicroRNA expression profile during adipogenic differentiation in mouse embryonic stem cells. Physiol Genomics 43: 611-620.

32. Gunaratne $\mathrm{PH}$ (2009) Embryonic stem cell microRNAs: defining factors in induced pluripotent (iPS) and cancer (CSC) stem cells? Curr Stem Cell Res Ther 4: 168-177.

33. Yang Z, Bian C, Zhou H, Huang S, Wang S, et al. (2011) MicroRNA hsamiR-138 inhibits adipogenic differentiation of human adipose tissue-derived mesenchymal stem cells through adenovirus EID-1. Stem Cells Dev 20: 259267.

34. Liu S, Yang Y, Wu J (2011) TNFît-induced up-regulation of miR-155 inhibits adipogenesis by down-regulating early adipogenic transcription factors. Biochem Biophys Res Commun 414: 618-624.

35. Karbiener M, Fischer C, Nowitsch S, Opriessnig P, Papak C, et al. (2009) microRNA miR-27b impairs human adipocyte differentiation and targets PPARgamma. Biochem Biophys Res Commun 390: 247-251.

36. Lin Q, Gao Z, Alarcon RM, Ye J, Yun Z (2009) A role of miR-27 in the regulation of adipogenesis. FEBS J 276: 2348-2358.

37. Lee EK, Lee MJ, Abdelmohsen K, Kim W, Kim MM, et al. (2011) miR-130 suppresses adipogenesis by inhibiting peroxisome proliferator-activated receptor gamma expression. Mol Cell Biol 31: 626-638.

38. Chan LS, Yue PY, Kok TW, Keung MH, Mak NK, et al. (2012) Ginsenoside$\mathrm{Rb} 1$ promotes adipogenesis through regulation of PPAR ${ }^{3}$ and microRNA-27b. Horm Metab Res 44: 819-824.

39. Kinoshita M, Ono K, Horie T, Nagao K, Nishi H, et al. (2010) Regulation of adipocyte differentiation by activation of serotonin (5-HT) receptors 5-HT2AR and 5-HT2CR and involvement of microRNA-448-mediated repression of KLF5. Mol Endocrinol 24: 1978-1987.

40. Kennell JA, Gerin I, MacDougald OA, Cadigan KM (2008) The microRNA miR-8 is a conserved negative regulator of Wnt signaling. Proc Natl Acad Sci U S A 105: 15417-15422.

41. Ross SE, Hemati N, Longo KA, Bennett CN, Lucas PC, et al. (2000) Inhibition of adipogenesis by Wnt signaling. Science 289: 950-953.

42. Liu Y, Zhang ZC, Qian SW, Zhang YY, Huang HY, et al. (2013) MicroRNA-140 promotes adipocyte lineage commitment of $\mathrm{C} 3 \mathrm{H} 10 \mathrm{~T} 1 / 2$ pluripotent stem cells via targeting osteopetrosis-associated transmembrane protein 1. J Biol Chem 288: $8222-8230$ 